\title{
Study on the Quality Control Chart for Determination of Soil Organic Carbon by Instrumental Analysis
}

\author{
Zhang Fuling ${ }^{1,2}$, Zhang Zhuo ${ }^{1,2,3}$, Diao Er-long ${ }^{1,2}$, Zhao Meiliang ${ }^{1,2}$, Liu Menglin ${ }^{1,2}$, Qiu Xunxun ${ }^{1,2}$ \\ ${ }^{1}$ Key Laboratory of Qinghai Province Physical Geography and Environmental Process, Qinghai Normal University, Qinghai Xining \\ 810008 , China \\ ${ }^{2}$ School of Life and Geography Sciences, Qinghai Normal University, Qinghai Xining810008, China \\ ${ }^{3}$ Environmental Monitoring Station of Kezilsu Prefecture, Xin jiang Atushi Xinjiang 845350, China
}

\begin{abstract}
Objective】 To ensure that each analysis step is in the monitoring state. The quality control chart is used to control the process of soil organic carbon content determination, and the reasons for drifting or exceeding the allowable value of the result data can be found out in time. 【Method】 The content of soil organic carbon in quality control samples was determined by instrumental analysis, and the quality control chart was drawn based on the determination data in Excel 2007, which was used for the quality control of the soil organic carbon content determination process. 【Result】 The control line of the mean control chart was $41.94 \% \sim 40.51 \%$, and the warning limit was $41.70 \% \sim 41.74 \%$. The control line range of the range control chart is $0.00 \% \sim 2.75 \%$, and the warning limit is $0.00 \% \sim 2.12 \%$. 【Conclusion】 The quality control chart method is simple to operate,easy to master, and can timely find the abnormal points or abnormal trends of data, which has high application value in test analysis, and can ensure the accuracy of laboratory test results.
\end{abstract}

\section{Introduction}

In laboratory, it is necessary to take a series of quality control and quality assurance measures of internal and external to ensure the precision and accuracy of test data, such as laboratory blank tests, parallel blank tests, plain code and security code parallel sample tests, quality control sample tests, sampling recovery rate tests, laboratory capability verification, and methods comparison etc., so as to assess whether the entire analysis process is in a "statistical control condition". Even under ideal analysis conditions, such as a tester testing the same batch of quality control samples with the same analysis test method in different time periods, the analysis results obtained are also inconsistent. There is a slight fluctuation around the truth value, that is, random errors are inevitable. However, according to mathematical statistics researches, if the fluctuation exceeds a certain range, the test result can be treated as an outlier. Therefore, the purpose for drawing the quality control chart is that: under the monitoring conditions, the analysis and test result should have the precision and accuracy in a certain range, and be a normal distribution (for example, [1]). Nowadays, the quality control chart is a method widely recommended by environmental monitoring, rock and mineral testing, agricultural environment testing, water quality testing, medical industry, etc., and its purpose is to ensure different kinds of analysis and testing processes are in a controlled state, so that the precision of test results and accuracy can be control within the preset control range (for example, [2, 3]). Walter Shewhart first proposed the quality control chart in 1928, he believed that, due to the influence of time and environment in sample analysis, no matter which method is used, there will be a certain degree of drift in the testing data, even under ideal conditions, random error will also occur, and then he proposed a statistical graph that can distinguish normal and abnormal fluctuations. In the long-term usage of the testing instrument, as the drift may cause the measurement result to exceed the allowable limit, the method proposed by Irfan Ertuğrul et al. (for example, [4]) that using control charts as a graphical analysis method are adopted. The quality control chart is a graphical analysis method with flexible form, appropriate mathematical description concept and more reasonable results, which can determine whether product quality is controlled in the acceptable limits range, and the signal will be sent when the product quality test results exceed the limit. Guo Aihua et al. (for example, [5]) proposed that the quality control chart is a method that draws the sample data point collected periodically in a process to a graph in sequence, of which purpose is to distinguish the quality variation in the detection process and find abnormal variations, and then adopt corrective and preventive measures to make the testing work smoothly going. Jia Yunhai et al. (for example, [6]) evaluated the long-term stability of testing instrument by using the standard deviation of the measurement results

* Corresponding author: sxzhangzhuo@126.com 
over a long period or the standard deviation of the average value measured at different periods. Some scholars conducted detailed research and analysis on the drawing and application of laboratory analysis quality control charts by using instance analysis methods. For example, Peng Nanlan et al. (for example, [7]) established a standard template through establishing quality control charts with Excel software, which can generate the corresponding quality control chart automatically by importing the original data, and it is very convenient for data store and statistic. When analyzed the residual pesticides in drinking water, Jiirgen Vogelgesang (for example, [8]) used various types of quality control charts to record the recovery rate of test, and it was found that the quality assurance system can help to understand the entire pesticide palette and how it changed over time. Huang Chunsha et al. (for example, [9]) established a method for drawing the $\mathrm{pH}$ value quality control chart of atmospheric precipitation in the acid rain observation station of Baise City, Guangxi; Tang Kun (for example, [10])clarified the methods and techniques for drawing the mean quality control chart and recovery quality control chart, and compared with other quality control charts, the quality control charts have the characteristics of simple calculation, clear graphics and effective control. Zuo Yi et al. (for example, [11]) used high-performance liquid chromatography to determine the content of metronidazole tablets in quality control samples. The measured data was drawn into a control chart and used for quality control of the metronidazole sample content determination process. Li Xinhong (for example, [12]) used the ammonia nitrogen determination process in water samples through the Nessler's reagent spectrophotometric method as an example to introduce the application of quality control charts in sample analysis and quality control. The previous research objects were mostly water quality, food, and medicine, and using instrumental analysis to test soil physical and chemical properties and to draw quality control charts has been rarely reported. Taking the soil on the southern slope of the Qilian Mountains in Qinghai as the research object, nearly 1500 soil samples were collected to successfully complete the study of the soil carbon and nitrogen content and migration mechanism in this area. And taking the process of soil organic carbon content as an example, through the quality control chart method, we first conducted a comprehensive evaluation of the measurement process to find abnormal points or abnormal trends and the cause in time. Using quality control charts for internal quality control has laid the foundation for accurate testing of soil element content and carbon storage and fixation in the southern slope of the Qilian Mountains in Qinghai.

\section{Materials and Methods}

\subsection{Materials}

The soil organic carbon was measured with the Vario EL III element analyzer produced by Elementar Ltd. in Germany, and the percentage content of $\mathrm{C}$ and $\mathrm{N}$ elements could be measured and calculated using the built-in workstation; the experiment used the Swiss-made electronic balance METTLER TOLEDO with a sensitivity of $0.001 \mathrm{mg}$. Quality control samples are provided by Analysensysteme GmbH, with batch number: 15.00-0062, and specification: $10.0 \mathrm{~g}$.

\subsection{Methods}

The C, H, N, S mode was selected, and the test method was $5 \mathrm{mg} / 90 \mathrm{~s}$. The measurement temperature was $1150^{\circ} \mathrm{C}$, the reduction temperature was $850{ }^{\circ} \mathrm{C}$. $\mathrm{O} 2$ pressure was $0.2 \mathrm{MPa}$, flow rate was $13 \mathrm{ml} / \mathrm{min}$. The He gas pressure was $0.12 \mathrm{MPa}$, and flow rate was $300 \mathrm{ml} / \mathrm{min}$.

Based on the test result data, we used Office Excel 2007 to insert the scatter line chart in the chart, drew the quality control chart, and connected the quasi-data preparation table with the scatter line chart, and then the corresponding quality control chart can be automatically generated.

\section{Results and analysis}

The quality control chart believes that there is a certain random error in the analysis process, and we can monitor and determine whether this random error is within a certain allowable range. In terms of probability theory and mathematical statistics, there is a $99 \%$ chance that the measurement result should be in the range of $\mathrm{X} \pm 3 \mathrm{~S}$ (upper and lower control lines); 95\% should be in the range of $\mathrm{X} \pm 2 \mathrm{~S}$ (upper and lower warning lines); $68 \%$ should be in the range of $\mathrm{X} \pm \mathrm{S}$ (upper and lower auxiliary lines). In general, according to the different test objects, there are mainly several quality control chart types such as mean quality control chart, mean-range quality control chart, and standard recovery quality control chart.

\subsection{Mean quality control chart}

Table 1. Original Data

\begin{tabular}{|c|c|c|c|c|}
\hline Number & Reault 1 & Reault 2 & Mean & Range \\
\hline 1 & 39.78 & 41.48 & 40.89 & 0.22 \\
\hline 2 & 41.45 & 40.35 & 40.90 & 0.50 \\
\hline 3 & 40.07 & 41.43 & 40.75 & 0.49 \\
\hline 4 & 41.25 & 41.55 & 41.40 & 0.58 \\
\hline 5 & 41.37 & 41.53 & 41.45 & 0.32 \\
\hline 6 & 40.67 & 41.45 & 41.06 & 0.20 \\
\hline 7 & 40.86 & 41.55 & 41.20 & 0.26 \\
\hline 8 & 41.03 & 41.57 & 41.30 & 0.18 \\
\hline 9 & 40.96 & 41.25 & 41.11 & 0.20 \\
\hline 10 & 41.04 & 41.93 & 41.49 & 0.28 \\
\hline 11 & 41.03 & 41.70 & 41.36 & 0.10 \\
\hline 12 & 41.06 & 41.79 & 41.43 & 0.28 \\
\hline 13 & 40.68 & 41.96 & 41.32 & 0.02 \\
\hline
\end{tabular}




\begin{tabular}{|l|l|l|l|l|}
\hline 14 & 40.20 & 41.80 & 41.00 & 0.32 \\
\hline 15 & 40.46 & 41.77 & 41.11 & 0.30 \\
\hline 16 & 40.48 & 41.65 & 41.06 & 0.38 \\
\hline 17 & 41.68 & 41.56 & 41.62 & 0.64 \\
\hline 18 & 40.60 & 41.67 & 41.13 & 0.44 \\
\hline 19 & 41.16 & 41.72 & 41.44 & 0.76 \\
\hline 20 & 41.22 & 41.76 & 41.49 & 0.19 \\
\hline
\end{tabular}

Based on the measurement results in Table 1, we can obtain:

-Mean value $X=41.23$, Standard deviation

$\mathrm{S}=0.24$, Center line $\mathrm{X}=41.23$;

-Upper auxiliary line $\mathrm{X}+\mathrm{S}=41.46$,

Lower auxiliary line $\mathrm{X}-\mathrm{S}=40.99$;

-Upper warning limit $X+2 S=41.70$,

Lower warning limit $\mathrm{X}-2 \mathrm{~S}=41.74$;

-Upper control limit $X+3 S=41.94$,

Lower control limit X-3S=40.51

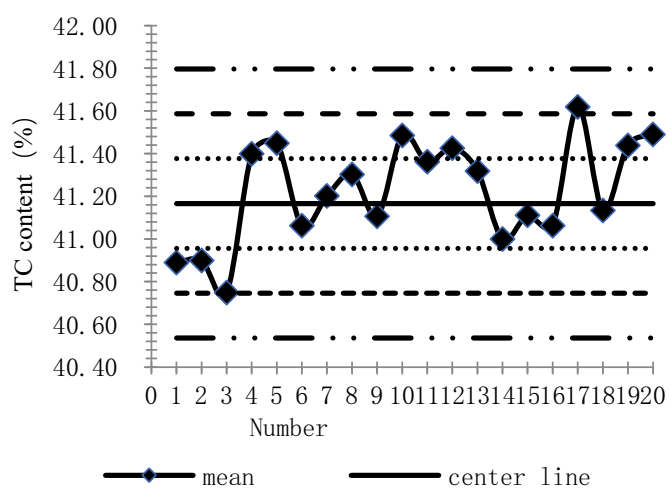

Fig1. The mean of quality control chart

\subsection{Mean-Range Quality Control Chart}

Factors such as A2 and D4 need to be introduced to produce the mean-range control chart. These factors are calculated values based on the number of repeated measurements and can be found from Table $3^{[2 \sim 3]}$.

Table 2. The coefficient of cuality control chart

\begin{tabular}{|c|c|c|c|}
\hline $\mathbf{n}$ & $\mathbf{A 2}$ & $\mathbf{D 3}$ & $\mathbf{D 4}$ \\
\hline 2 & 1.88 & 0 & 3.27 \\
\hline 3 & 1.02 & 0 & 2.58 \\
\hline 4 & 0.73 & 0 & 2.28 \\
\hline 5 & 0.58 & 0 & 2.12 \\
\hline 6 & 0.48 & 0 & 2 \\
\hline 7 & 0.42 & 0.076 & 1.92 \\
\hline 8 & 0.37 & 0.136 & 1.86 \\
\hline
\end{tabular}

$\mathrm{n}$ represents the number of parallel samples in each measurement In the mean control chart:

-Mean $\mathrm{X}=41.23$, Mean Range $\mathrm{R}=0.84$;

-Upper auxiliary line $X+1 / 3$ A2R $=41.75$, Lower auxiliary line $\mathrm{X}-1 / 3 \mathrm{~A} 2 \mathrm{R}=40.70$;

-Upper cordon $X+2 / 3 \mathrm{~A} 2 \mathrm{R}=42.28$, Lower cordon $\mathrm{X}$ 2/3 A2R $=40.17$
-Upper control line $\mathrm{X}+\mathrm{A} 2 \mathrm{R}=42.81$, Lower control line $\mathrm{X}-\mathrm{A} 2 \mathrm{R}=39.64$.

In the range control chart:

-The center line is the average range $\mathrm{R}=0.84$

-Upper auxiliary line $\mathrm{R}+1 / 3(\mathrm{D} 4 \mathrm{R}-\mathrm{R})=1.48$, Upper warning line $\mathrm{R}+2 / 3(\mathrm{D} 4 \mathrm{R}-\mathrm{R})=2.12$;

-Upper control line $\mathrm{D} 4 \mathrm{R}=3.27 \times 0.84=2.75$, Lower control line $\mathrm{D} 3 \mathrm{R}=0 \times 0.84=0.00$.
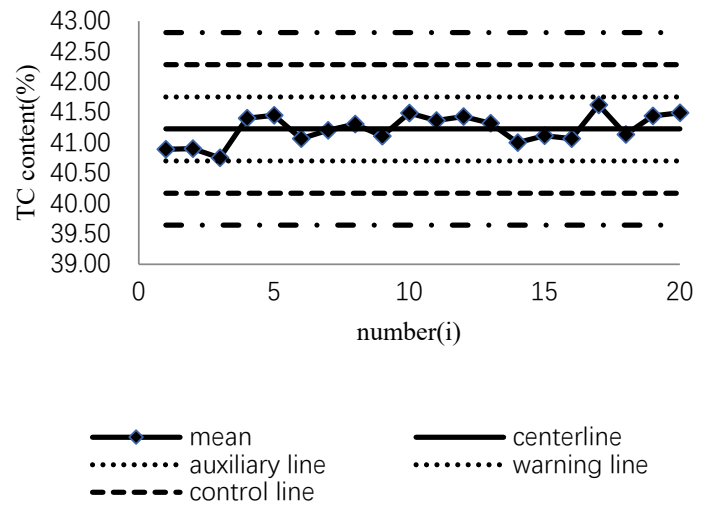

Fig2. Mean quality control chart section
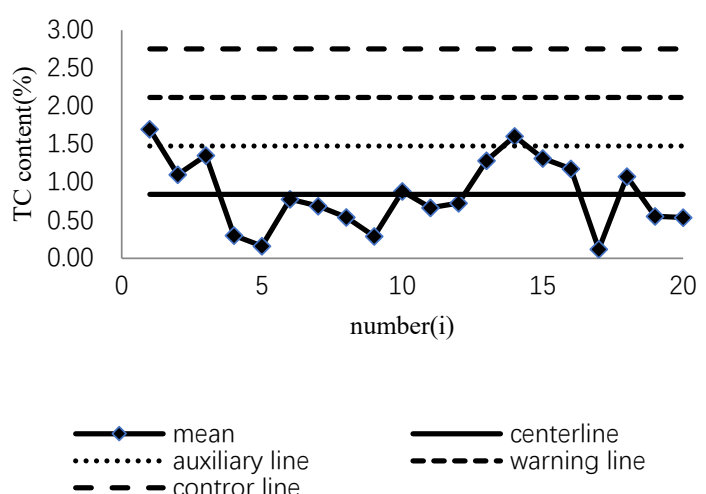

Fig3. Range quality control chart section

\subsection{Quality control chart of sampling recovery rate}

In the laboratory, the sampling recovery rate of the test components is often measured to ensure the accuracy of the analysis results. The amount of standard sample added should be as close as possible to the content of the unknown testing sample components, at most, less than three times of the unknown testing sample components content. When the content of the component in the sample is less than the lower limit of detection, the standard sample should add according to the detection limit. The calculation method for the sampling recovery rate is shown as:

Sampling recovery rate $=[(\mathrm{C} 2-\mathrm{C} 1) / \mathrm{C} 0] \times 100 \%$

-C2: The measured value of the unknown sample component after adding the standard sample;

$-\mathrm{C} 1$ : The measured value of unknown sample component;

-C0: The addition amount of standard sample. 
The method is like above preparation work before drawing the quality control chart. After accumulated 20 values of the sampling recovery rate, their mean and standard deviation can be obtained. With the mean value as the center line, the sum of mean value and the three times standard deviation as the upper control limit, and their difference as the lower control limit, the quality control chart of the sampling recovery rate can be drawn.

\section{Discussion and conclusion}

According to the "Conventional Quality Control Chart" (GB/T4091-2001)(for example, [13]) issued by the National Quality and Technical Supervision Bureau, the first step is to map the test results of the quality control samples in the order of testing to ensure that all points fall in the upper and lower control line. The second step is to check the position and the proportion of investment points. If they satisfy the eight criteria such as 15 consecutive points within the upper and lower auxiliary limits on both sides of the center line, 8 consecutive points are on both sides of the center line but no point falls within the upper and lower auxiliary limit, it is indicated that the distribution of points is very unreasonable. If the above situation occurs, the reasons should be identified, the untrustworthy data points should be eliminated, and the quality control samples should be re-tested until the test requirements are met. The quality control points made based on the measurement order of soil organic carbon content are all within the monitoring range, the distribution is reasonable, and there are no abnormal points or abnormal trends.

Before testing unknown samples, the analysis and testing personnel should first determine the quality control samples in accordance with different quality control measures, draw a quality control chart, and clearly control the upper and lower limits. The quality control samples issued by the quality control room must be analysed at the same time as the unknown samples to be tested. After the test completed, the result data is immediately placed on the quality control chart. If the setting point is inside the control lines, it proves that the process is under monitoring state and the results are reliable. If it is outside the upper and lower control lines, it proves that the test results have deviations. In the determination of soil physical and chemical properties, the quality control chart is a preferred internal quality control method. Compared with other quality control methods, data preparation forms is directly linked to the scatter line icon using Office Excel 2007, the quality control chart method can directly reduce the drawing difficulty and improve the drawing speed, which can be used as a standard template, and is very conducive to the calculation of test result data statistics. The quality control chart is simple and practical to draw and use. Analysts can find abnormalities in the test process in time through the quality control charts, comprehensively consider the causes of abnormal environments, personnel, instruments, operations, etc., and take a series of quality control measures to eliminate or make additional measurements and re-test abnormal points to ensure the accuracy of the result data.
Therefore, for the inspection and testing agencies analysis and testing personnel no matter in agricultural environment testing, rock and mineral testing, environmental monitoring or other, it is necessary to continuously research and summary of the above various types of quality control charts, comprehensive comparison and use them. And combined the method of random sampling and retest in the laboratory, the accuracy and comparability of the analysis results of the whole sample are guaranteed to judge whether the measurement process is affected by uncontrolled system effects or random effects. The analysis quality of each group of samples is controlled, and quality control charts should be flexibly applied in the whole process of relevant test analysis and control.

\section{References}

1. Zhao Xingkai,Li Zengyao,Zhu Qingke,Zhu Danhong,Liu Huifang. Climatic and drought characteristics in the loess hilly-gully region of China from 1957 to 2014.[J]. PloS one,2017,12(6)

2. Edited by China Environmental Monitoring Centre. Environmental Water Quality Monitoring Quality Assurance Manual [M]. Beijing: Chemical Industry Press, 2010.

3. Edited by China National Accreditation Board of Laboratory;Basic knowledge of laboratory accreditation and management [M]. Beijing: China Metrology Press, 2001.

4. Irfan Ertuğrul, Mustafa Güneş. The Usage of Fuzzy Quality Control Charts to Evaluate Product Quality and an Application[M]. Analysis and Design of Intelligent Systems using Soft Computing Techniques. Springer Berlin Heidelberg, 2007.

5. Guo Aihua, Li Ye, Wang Wei. Quality Control of Test Results of Chemical Analysis Laboratory [J]. Physical and Chemical Inspection (Chemistry), 2015, 51 (04): 528-531.

6. Jia Yunhai, Sun Xiaofei, Yuan Liangjing.Research on Characterization Method of Long-term Stability of Analytical Instruments[J] .Metallurgical Analysis, 2019,39 (01): 1-7.

7. Peng Nanlan, Tian Jianping, Wang Xuewei, Tang Qiping, Tan Fei.Computer production of quality control maps in geological and mineral laboratories [J] .Yunnan Geology, 2013,32 (01): 72-75.

8. Jürgen Vogelgesang. The quality control chart principle: application to the routine analysis of pesticide residues in water[J]. Fresenius' Journal of Analytical Chemistry, 1991, 340(6):384-388.

9. Huang Chunsha, Zhu Xiaoyan .The use of Excel to make acid rain $\mathrm{pH}$ quality control chart $[\mathrm{J}]$. Guizhou Meteorology, 2013,37 (04): 52-54.

10. Tang Kun.The production and application of laboratory analysis quality control chart $[\mathrm{J}]$.Journal of Anhui Agricultural Sciences, 2004 (01): 200-202. 
11. Zuo Yi, Fei Luhua.Application of quality control chart in the determination of metronidazole tablets [J] .Medical Herald, 2018,37 (12): 1498-1499.

12. Li Xinhong. Application of quality control chart in quality control of environmental analysis [J]. Guangzhou Environmental Science, 2008 (02): 24-25.

13. GB / T 4091-2001Routine quality control chart [S]. 